\title{
BMJ Open Regional citrate versus systemic heparin anticoagulation for continuous renal replacement therapy in critically ill patients with acute kidney injury (RICH) trial: study protocol for a multicentre, randomised controlled trial
}

\author{
Melanie Meersch, ${ }^{1}$ Mira Küllmar, ${ }^{1}$ Carola Wempe, ${ }^{1}$ Detlef Kindgen-Milles, ${ }^{2}$ \\ Stefan Kluge, ${ }^{3}$ Torsten Slowinski, ${ }^{4}$ Gernot Marx, ${ }^{5}$ Joachim Gerss, ${ }^{6}$ \\ Alexander Zarbock, ${ }^{1}$ SepNet Critical Care Trials Group
}

To cite: Meersch M, Küllmar M, Wempe $\mathrm{C}$, et al. Regional citrate versus systemic heparin anticoagulation for continuous renal replacement therapy in critically ill patients with acute kidney injury (RICH) trial: study protocol for a multicentre, randomised controlled trial. BMJ Open 2019;9:e024411. doi:10.1136/ bmjopen-2018-024411

- Prepublication history and additional material for this paper are available online. To view please visit the journal (http:// dx.doi.org/10.1136/bmjopen2018-024411).

$\mathrm{JG}$ and $\mathrm{AZ}$ contributed equally. MM and MK contributed equally.

Received 24 May 2018

Revised 24 0ctober 2018

Accepted 5 November 2018

Check for updates

(C) Author(s) (or their employer(s)) 2019. Re-use permitted under CC BY-NC. No commercial re-use. See rights and permissions. Published by BMJ.

For numbered affiliations see end of article.

Correspondence to Professor Alexander Zarbock; zarbock@uni-muenster.de

\section{ABSTRACT}

Introduction Acute kidney injury (AKI) is a wellrecognised complication of critical illness which is of crucial importance for morbidity, mortality and health resource utilisation. Renal replacement therapy (RRT) inevitably entails an escalation of treatment complexity and increases costs for those patients with severe AKI. However, it is still not clear whether regional citrate anticoagulation or systemic heparin anticoagulation for continuous RRT (CRRT) is most appropriate. We hypothesise that, in contrast to systemic heparin anticoagulation, regional citrate anticoagulation for CRRT prolongs filter life span and improves overall survival in a 90-day follow-up period (coprimary endpoints).

Methods and analysis We will conduct a prospective, randomised, multicentre, clinical trial including up to 1450 critically ill patients with AKI requiring CRRT. We suggest to investigate the effect of regional citrate anticoagulation for CRRT as compared with systemic heparin anticoagulation. The two coprimary outcomes are filter life span and overall survival in a 90-day follow-up period. Secondary outcomes are length of stay in the intensive care unit; length of hospitalisation; duration of CRRT; recovery of renal function at days $28,60,90$ and 1 year; requirement for RRT after days 28, 60, 90 and 1 year; 28 days, 60 days, 90 days and 1 -year all-cause mortality; major adverse kidney events at days 28, 60, 90 and 1 year; bleeding complications; transfusion requirements; infection rate and costs of RRT. Additionally, in an add-on study involving several of the participating centres, blood samples from recruited patients will be collected at different time points to analyse whether the anticoagulation strategy has an impact on immune response as evidenced by leucocyte recruitment and function.

Ethics and dissemination The RICH trial has been approved by the Federal Institute for Drugs and Medical Devices, the leading Ethics Committee of the University of Münster and the corresponding Ethics Committee at each participating site.

Trial registration number NCT02669589.
Strengths and limitations of this study

- The trial will be conducted as large randomised controlled, multicentre, two-arm, parallel-group trial investigating the two routinely used anticoagulation strategies regional citrate anticoagulation and systemic heparin anticoagulation for renal replacement therapy in critically ill patients; the results of this trial will improve the care of critically ill patients with acute kidney injury and allow to give more precise recommendations in future guidelines on acute kidney injury.

- The use of a combined endpoint consisting of filter life span and mortality will evaluate an objective statement of the process of continuous renal replacement therapy together with a patient-centred outcome.

- The trial has several strengths: a specific protocol for a uniform initiation of renal replacement therapy, a standardised continuous renal replacement therapy treatment according to the Kidney Disease: Improving Global Outcomes guidelines, the patient population (patients with sepsis and other critical conditions such as haemodynamic instability with high dose of vasopressor support and refractory fluid overload) and the multicentre trial design together with the large patient cohort of surgical as well as non-surgical patients.

- An add-on study will investigate the host immune response during regional citrate anticoagulation and systemic heparin anticoagulation since some preliminary studies have suggested that the choice of anticoagulant influences the levels of proinflammatory mediators and leucocyte function.

- The lack of blinding of the investigator and the selection bias (because patients requiring a therapeutic anticoagulation or having contraindications against one of the anticoagulants cannot be included in the study protocol) are limitations of the study design. 


\section{INTRODUCTION}

Acute kidney injury (AKI) is a common complication occurring in up to $50 \%$ of critically ill patients. ${ }^{1}$ The mortality rate reaches up to $60 \%{ }^{2}$ As patients die of AKI and not simply with, AKI represents a specific and independent risk factor for poor outcome. ${ }^{34}$ The treatment of AKI remains primarily supportive, with renal replacement therapy (RRT) being the gold standard for severe AKI. Many key issues regarding the optimal management of RRT are still a focus of controversy. In the critical care setting, continuous RRT (CRRT) is currently preferred over intermittent techniques in an attempt to ensure haemodynamic stability, tight volume control and acid-base balance. A major disadvantage of CRRT is the need for continuous anticoagulation to prevent clotting of the extracorporeal circuit and thromboembolic complications. In clinical practice, systemic anticoagulation with heparin (SAH) and regional anticoagulation with citrate (RCA) are the two main anticoagulation strategies for CRRT. However, it still remains unknown whether SAH or RCA is equivalent in terms of filter life span and patients-centred outcomes such as morbidity and mortality.

In terms of filter life span, Kutsogiannis et alperformed a randomized controlled trial (RCT) in 30 critically ill patients undergoing CRRT with RCA or SAH. A total of 79 haemofilters were analysed. Filter life span was significantly longer with RCA as compared with SAH (124.5 hours vs 38.3 hours; $\mathrm{p}<0.001$ ). Similar results were shown in a recently published trial analysing 857 study circuits comparing RCA/calcium anticoagulation and regional heparin/protamine anticoagulation (390 in the citrate and 467 in the heparin group). ${ }^{5}$ Circuit clotting was more likely in the heparin than in the citrate group (HR 2.03; 95\% CI 1.36 to 3.03 ; p<0.0005). A recently published meta-analysis including 11 RCTs showed less circuit loss (HR 0.76; 95\% CI 0.59 to $0.98 ; \mathrm{p}=0.04$ ) and less filter failure (RR $0.70 ; 95 \%$ CI 0.50 to $0.98 ; \mathrm{p}=0.04$ ) in the citrate group as compared with the heparin group. ${ }^{6}$

In terms of mortality, Hetzel et al demonstrated in a multicentre trial with 174 patients that mortality rates per day were similar between the two groups during both the treatment and follow-up periods $(3.1 \%$ vs $3.1 \%$ and $3.8 \%$ vs $3.4 \%$, respectively). ${ }^{7}$ In contrast, Oudemans-van Straaten et al demonstrated in a single-centre trial that RCA reduced both hospital and 90-day mortality by $18 \%$ $(p=0.02) .{ }^{8}$ The authors suggested that these beneficial effects may result from the immunomodulatory effects of citrate. Schilder et al intended to perform a large multicentre randomised controlled trial (RCT) to investigate the effects of RCA on 28-day mortality. ${ }^{9}$ However, after enrolling 139 patients, the trial was discontinued as a result of slow recruitment process. Two meta-analyses, including data from six small and underpowered RCTs, suggested that RCA significantly reduces the risk of bleeding. ${ }^{10} 11$

However, data are inconclusive and large RCTs are missing to arrive at a definitive conclusion. On this account, we will perform a large national, multicentre, RCT to increase evidence whether the use of RCA prolongs filter life span and overall survival in a 90-day follow-up period compared with SAH.

\section{OBJECTIVES AND AIMS}

Aim 1

To compare the clinical effectiveness of RCA and SAH in critically ill patients with AKI undergoing CRRT, we are testing the following:

- Hypothesis I: RCA as compared with SAH for CRRT in critically ill patients prolongs filter life span and overall survival, resulting in a reduction of 90-day all-cause mortality by approximately $8 \%$ (from $48 \%$ to $40 \%$ ).

\section{Aim 2}

To understand whether the different anticoagulation strategies in patients undergoing CRRT affect mechanisms of illness and recovery, we are testing the following:

- Hypothesis II: RCA and SAH show different biomarker and cytokine expression.

\section{Aim 3}

We aim to assess the costs and cost-effectiveness of the different anticoagulation strategies for CRRT.

\section{METHODS AND ANALYSIS Design and setting}

The 'Regional citrate versus systemic heparin anticoagulation for continuous renal replacement therapy in critically ill patients with acute kidney injury (RICH)' trial is a randomised, multicentre, two-arm, parallel-group trial conducted at 31 centres across Germany (online supplementary table 1). The trial protocol design follows Consolidated Standards of Reporting Trials and the conduct of the study follows the Declaration of Helsinki (version Fortaleza, 2010). The flow chart is summarised in figure 1 .

\section{Patient and public involvement}

Patients and public were not involved in the research design of the study. However, study results will be published open access. If desired, patients or their representatives can be informed through a brief summary of the results distributed by the local investigators.

\section{Participants}

Eligible patients need to fulfil all inclusion and none of the exclusion criteria. The inclusion criteria are designed to identify critically ill patients with severe AKI who need CRRT. All five inclusion criteria must be fulfilled at the time of screening: (1) severe AKI (Kidney Disease: Improving Global Outcomes (KDIGO) 3 classification) despite optimal resuscitation or absolute indication for CRRT, (2) at least one additional condition (sepsis or septic shock, ${ }^{12}$ use of high vasopressor doses, refractory 


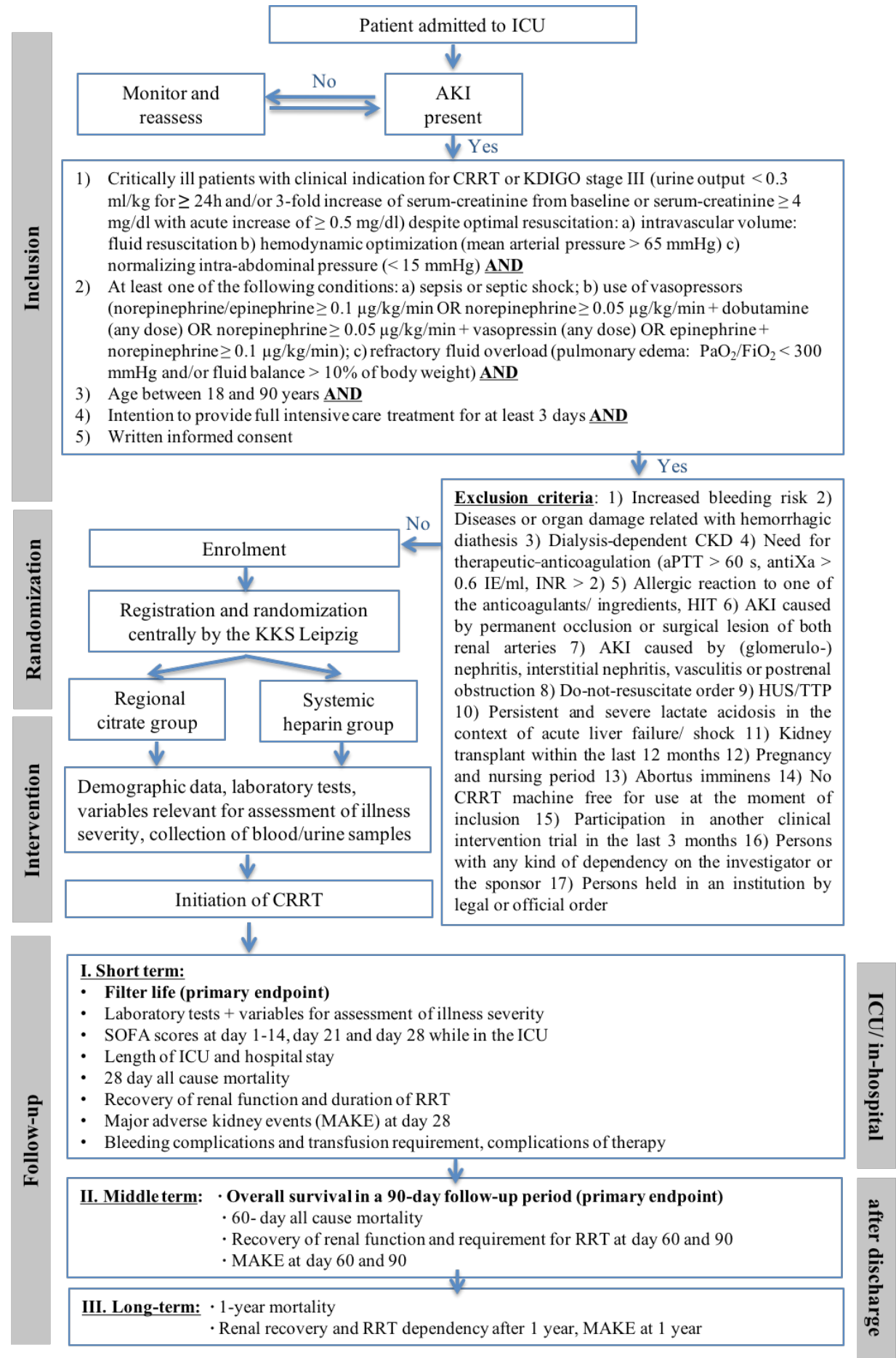

Figure 1 Trial workflow. The research coordinators will screen patients in all participating ICUs for eligibility on a daily basis. Prior to enrolment, it is assured that fluid status is optimised if necessary. Patients not yet fulfilling the inclusion criteria will be rescreened each day. Patients fulfilling one of the exclusion criteria will be excluded and not rescreened. Before initiating RRT, blood and urine samples will be collected and different variables will be documented. CRRT will be started as soon as possible in patients with a clinical indication for RRT or within 24 hours after diagnosing severe AKI (KDIGO stage 3 ). Patients in the 'regional citrate group' receive regional citrate with a posthaemofilter ionised $\mathrm{Ca}^{++}$level of $0.25-0.35 \mathrm{mmol} / \mathrm{L}$ as anticoagulant for CRRT. Patients in the 'systemic heparin group' receive systemic heparin with a target aPTT of 45-60s as anticoagulant for CRRT. Laboratory tests will be analysed and variables relevant for the assessment of illness severity will be recorded during ICU stay on days 1-14, day 21, day 28. Follow-up will be performed after days 60, 90 and 1 year. AKI, acute kidney injury; aPTT, activated Partial Thromplastin Time; CKD, chronic kidney disease; CRRT, continuous renal replacement therapy; $\mathrm{FiO}_{2}$, fractional inspired oxygen; HIT, heparin-induced thrombocytopaenia; HUS, haemolytic uraemic syndrome; ICU, intensive care unit; INR, International Normalized Ratio; KDIGO, Kidney Disease: Improving Global Outcomes; KKS, Koordinierungszentrum für Klinische Studien (coordination center for clinical trials); $\mathrm{PaO}_{2}$, arterial oxygen tension; RRT, renal replacement therapy; TTP, thrombotic thrombocytopaenic purpura. 
Table 1 Inclusion and exclusion criteria

Inclusion

Exclusion

$\mathrm{AKI}$, acute kidney injury; aPTT, activated partial thromboplastin time; CRRT, continuous renal replacement therapy; $\mathrm{FiO}_{2}$, fractional inspired oxygen; INR, international normalized ratio; KDIGO, Kidney Disease: Improving Global Outcomes; $\mathrm{PaO}_{2}$, arterial oxygen tension.

fluid overload), (3) age between 18 and 90, (4) intention to provide full intensive care treatment for at least 3 days and (5) written informed consent (table 1).

Taking into consideration the feedback from the different participating sites and the slow randomisation process, we adjusted the exclusion criteria and modified the protocol in September 2017 (online supplementary table 2).
1. Severe AKI (KDIGO 3 classification) despite optimal resuscitation

- Urine output of $<0.3 \mathrm{~mL} / \mathrm{kg} / \mathrm{hour}$ for $\geq 24$ hours.

- Less than threefold increase in serum creatinine level compared with the baseline value. OR

Critically ill patients with an absolute clinical indication for CRRT

- Urea serum levels $>150 \mathrm{mg} / \mathrm{dL}$.

- Potassium serum levels $>6 \mathrm{mmol} / \mathrm{L}$.

Magnesium serum levels $>4 \mathrm{mmol} / \mathrm{L}$

Urine production $<200 \mathrm{~mL} / 12$ hours or anuria.

Organ oedema in the presence of AKI resistant to diuretic treatment.

At least one of the following conditions $\mathrm{kg} / \mathrm{min}+$ dobutamine (any dose) or norepinephrine $\geq 0.05 \mu \mathrm{g} / \mathrm{kg} / \mathrm{min}+\mathrm{vasopressin}$ (any dose) or epinephrine+norepinephrine $\geq 0.1 \mu \mathrm{g} / \mathrm{kg} / \mathrm{min}$ ).

$>10 \%$ of body weight).

3. Age between 18 and 90 .

4. Intention to provide full intensive care treatment for at least 3 days.

5. Written informed consent of the patient or his legal representatives or the authorised representative or inclusion due to an emergency situation. gastrointestinal tract, hypertension with a diastolic blood pressure $>105 \mathrm{~mm} \mathrm{Hg}$, intracranial haemorrhage or injuries (intracranial haemorrhage, aneurysm of brain arteries) or surgical procedures on the central ervous system (if according to neurologists or neurosurgeons a heparinisation with target aPTT of 45-60 active tuberculosis, infective endocarditis).

2. Diseases or organ damage related to haemorrhagic diathesis (coagulopathy, thrombocytopaenia, severe liver or pancreas disease).

3. Dialysis-dependent chronic kidney insufficiency.

4. Need of therapeutic anticoagulation (aPTT $>60 \mathrm{~s}$, anti-Xa $>0.6 \mathrm{IE} / \mathrm{mL}$, INR $>2$ ).

Allergic reaction to one of the anticoagulants, ingredients or a known Heparin-induced thrombocytopaenia

6. AKI caused by permanent occlusion or surgical lesion of both renal arteries.

7. AKI caused by glomerulonephritis, interstitial nephritis, vasculitis or urinary tract obstruction.

8. Do-not-resuscitate order.

9. Haemolytic uraemic syndrome/thrombotic thrombocytopaenic purpura.

10. Persistent and severe lactate acidosis in the context of acute liver failure and/or shock.

11. Kidney transplant within the last 12 months.

12. Pregnancy and nursing period (female patients must be surgically sterile or postmenopausal for at least 2 years; or, if of childbearing potential, negative serum pregnancy test (due to intensive care treatment and severity of illness, sexual abstinence is warranted).

13. Abortus imminens.

No machine for CRRT free for use at the moment of inclusion

16. Persons with any kind of dependency on the investigator or employed by the sponsor or investigator.

17. Persons held in an institution by legal or official order. 
written consent, the legally authorised representative will be asked. If there is no authorised representative, each centre is required to follow the recommendations of the local institutional review boards (IRBs). In this case, informed consent will be obtained as soon as possible by the patient or the legally authorised representative-as soon as the patient's condition allows it or as soon as a representative is available.

\section{Randomisation process}

Randomisation will be performed centrally by the Clinical Trials Centre Leipzig in a 1:1 proportion using a minimisation method with a random component. ${ }^{13}$ Randomisation will minimise the imbalance between the number of patients in the two treatment groups over the factors site, Cardiovascular Sequential Organ Failure Assessment (SOFA) Score (0-2 vs 3-4), presence or absence of oliguria and gender.

Treatment assignment will be accomplished using an internet-based randomisation tool. Patients will enter the treatment protocol immediately after randomisation.

\section{Management of RRT}

To ensure uniformity of treatment among sites and between both treatment groups, it is critical that specific protocols for the performance of RRT are strictly adhered to. All patients will be treated with CRRT. In patients with KDIGO stage 3 (table 1), CRRT needs to be started within 24 hours after meeting KDIGO stage 3 criteria. In patients with an absolute indication (table 1), CRRT needs to be initiated as soon as possible. The actually administered dialysis dose ranges from 20 to $25 \mathrm{~mL} /$ $\mathrm{kg} /$ hour. The prescribed dose is $30 \mathrm{~mL} / \mathrm{kg} /$ hour. Blood flow will be kept above $100 \mathrm{~mL} / \mathrm{min}$. The delivered dose of CRRT will be monitored. Filters need to be changed every 72 hours (according to the recommendations of the manufacturer).

\section{Treatment arms}

Randomised anticoagulation strategies will be performed as follows:

RCA: target $\mathrm{iCa}^{2+} 0.25-0.35 \mathrm{mmol} / \mathrm{L}$ (posthaemofilter).

SAH: target activated partial thromboplastin time (aPTT) of 45-60s.

\section{Cessation of RRT}

RRT can be discontinued if renal recovery defined by urinary output (UO) occurs (UO $>400 \mathrm{~mL} / 24$ hours without diuretics or UO $>2100 \mathrm{~mL} / 24$ hours with diuretics $\left.^{14}\right)$.

If cessation criteria are not fulfilled, CRRT will be performed for at least 5 days. After this time, the treating intensivist can switch to an intermittent RRT technique.

In the case of restart of CRRT during the index hospitalisation, the patient receives the previously randomised anticoagulation strategy.

\section{Outcomes}

The two coprimary outcomes of the study are (1a) filter life span and (1b) overall survival in a 90-day follow-up period.

Secondary outcomes include:

- Intensive care unit (ICU) and hospital length of stay.

- Duration and complication of RRT.

- Bleeding complications and transfusion requirement.

- Rate of infection during index ICU stay.

- Renal recovery (complete recovery: serum creatinine $\leq 0.5 \mathrm{mg} / \mathrm{dL}$ than baseline; partial recovery: serum creatinine $>0.5 \mathrm{mg} / \mathrm{dL}$ than baseline but not dialysis dependent; non-recovery: patients who remained dialysis dependent) at day 28, 60, 90 and 1 year.

- Need for RRT at day 28, 60, 90 and 1 year.

- All-cause mortality at day 28, 60, 90 and 1 year.

- Major adverse kidney events (defined as the composite of death, use of RRT and persistent renal dysfunction (defined as serum creatinine $>0.5 \mathrm{mg} / \mathrm{dL}$ than baseline or RRT dependency)) at day 28, 60, 90 and 1 year.

- Different proinflammatory and anti-inflammatory mediators (eg, interleukin (IL)-6, IL-8 and IL-10) will be compared across both treatment arms

\section{Sample size}

Power calculations were performed based on the two coprimary outcomes (1a) filter life span and (1b) overall survival in a 90-day follow-up period. The primary effectiveness analysis is intended to show a superiority of RCA versus SAH for CRRT in intensive care patients with AKI.

An adaptive design with one interim analysis has been established. The multiple (two-sided) significance level was set to alpha $=0.05$. The mean difference of filter life span between the treatment groups based on published data is expected to be at least 5 hours in favour of the RCA group ( \pm 27 hours SD within each group).${ }^{10}$ Overall survival is expected to follow an exponential distribution. The expected 90-day mortality rate in the SAH group is $48 \%$ based on recently published multicentre trials investigating the same patient population. ${ }^{7-9}$ Differences between treatment groups are considered to be clinically meaningful, if the 90-day mortality rate in the RCA group is $40 \%$ or lower. Follow-up of each patient will be 90 days. During this period, $10 \%$ of living patients are expected to be lost to follow-up. The corresponding process is expected to follow an exponential distribution. The required power regarding the first and second primary outcome was set to $90 \%$ and $80 \%$, respectively. This corresponds to a $70 \%$ power that both coprimary outcomes reach a significant result. The time points of the interim and final statistical analysis are determined from the first primary outcome. Resulting from these considerations, the interim analysis is performed when 400 patients have been recruited in total across both treatment groups and primary outcome data are available. The final analysis is intended to be performed when 1260 patients have been recruited. In the interim analysis, the sample size of the final analysis will be recalculated under the restriction of 
a maximal total number of 1450 patients. Power calculations were performed using the ADDPLAN software.

\section{Statistical analysis}

Statistical analyses will be performed according to the principles of the ICH guideline E9 'Statistical Principles for Clinical Trials' using standard statistical software (SAS, SPSS, ADDPLAN).

The randomised groups will be compared on all baseline variables using descriptive summary statistics such as mean and SD, median and quartiles or absolute and relative frequency, as appropriate.

An adaptive design with one interim analysis based on a group sequential plan according to O'Brien/Fleming is established. The time point of the interim analysis is determined from the first coprimary outcome filter life span. The interim analysis is conducted at the time when 400 patients have been recruited in total across both treatment groups and primary outcome data are available (information rate 0.5$)$. Regarding the first coprimary outcome, no futility stop is admitted. Regarding the second coprimary outcome overall survival, the trial may be stopped for futility (non-binding), if in the interim analysis the local $\mathrm{p}$ value of favourable survival in the RCA group is 0.5 or larger, or if stochastic curtailment shows a conditional power of the final statistical analysis with 1450 patients that is lower than $50 \%$. In the event of important new discoveries, the design of the study may be changed. In particular, the sample size of the final analysis will be recalculated.

The treatment effect on the first coprimary outcome filter life span will be evaluated using a (two-sided) inverse normal Likelihood Ratio test based on a multivariable linear mixed model. The treatment effect on the second coprimary outcome overall survival will be evaluated using a (two-sided) inverse normal Likelihood Ratio test based on a multivariable Cox regression model. Both Likelihood Ratio tests will be performed by building a null model with the factors study center, cardiovascular SOFA Score (0-2 vs 3-4), presence or absence of oliguria, and gender. An additional factor in the null model accounts for the changes of inclusion/ exclusion criteria that were implemented via amendment 1 . The first factor level indicates patients that were recruited before amendment 1 has been implemented and the second factor level indicates patients that were recruited after implementation of amendment 1 . The linear mixed model of the first coprimary outcome filter life span additionally includes a subject-specific random effect. The Likelihood Ratio tests are performed by comparing the null model to a model that additionally includes a treatment effect (RCA vs SAH).

The multiple (two-sided) significance level is set to alpha $=0.05$. In order to account for multiplicity due to the definition of two coprimary outcomes, a multiple testing procedure with fixed a priori ordered hypotheses is applied, that controls the familywise type I error in the strong sense according to Bauer. ${ }^{15}$ First, the null hypothesis of equal filter life span in both treatment groups is tested on a (two-sided) significance level alpha $=0.05$. If and only if this null hypothesis is rejected, subsequently the null hypothesis of equal overall survival is tested on a (two-sided) significance level alpha $=0.05$. Each of the above two-sided hypotheses is decomposed into two one-sided hypotheses on significance level alpha $=0.025$, respectively. The primary effectiveness analysis provides confirmatory statistical evidence.

Due to the fixed order of the tested hypotheses there are three possible results of our study. The difference of RCA as compared with SAH may prove to be

1. Not statistically significant with respect to both coprimary outcomes.

2. Statistically significant with respect to both coprimary outcomes.

3. Statistically significant with respect to the first primary outcome filter life, but not statistically significant with respect to the second primary outcome overall survival.

Our interpretation of the different possible results of our study will be:

1. There is no treatment effect on either coprimary outcome.

2. The use of RCA as compared with SAH prolongs filter life span and overall survival.

3. The use of RCA as compared with SAH prolongs filter life span, but the treatment effect is not large enough to result in an increased overall survival.

If the applied (two-sided) inverse normal Likelihood Ratio test shows a significant treatment effect on overall survival, the treatment effect will be estimated by means of the 90-day all-cause mortality rate in both treatment groups.

The primary effectiveness analysis will be performed according to the intention-to-treat principle (ITT) using the full analysis set of all randomised patients. Beyond the primary ITT analysis of the primary outcomes, sensitivity analyses will be performed, including per-protocol analyses.

Statistical analysis of prespecified secondary outcomes will be performed with descriptive and inferential statistical methods. The impact of transfusion requirements on survival will be evaluated using Cox regression with transfusion requirement as a time-dependent covariate. In subgroup analyses, surgical and conservatively treated patients will be analysed separately. Moreover, the use of predilution and postdilution method will be analysed separately for filter life span. Additional exploratory analyses will include safety analyses (including metabolic and anticoagulatory profiles, adverse events, serious adverse events). Results are generally reported by mean parameter estimates and associated 95\% CIs. All applied hypothesis tests will be two sided. Missing values that may arise in effectiveness or safety parameters will not be replaced by any kind of statistical imputation.

\section{TRIAL MANAGEMENT}

\section{Safety}

Adverse events are defined according to the Directive 2001/20/EC, the European Detailed Guidance CT 3, 
Table 2 Reportable adverse events

\begin{tabular}{|c|c|c|}
\hline \multicolumn{2}{|l|}{ Adverse events } & \multirow[b]{2}{*}{ Serious adverse events } \\
\hline Disease related & CVC and renal replacement therapy (RRT) related & \\
\hline $\begin{array}{l}\text { Death caused by underlying } \\
\text { diseases (eg, severe sepsis/ } \\
\text { septic shock). } \\
\text { Cardiovascular events: } \\
\text { aggravation of known congestive } \\
\text { heart failure, new myocardial } \\
\text { infarction after known acute } \\
\text { myocardial infarction. } \\
\text { Neurological events: aggravation } \\
\text { of intracerebral bleeding, rupture } \\
\text { of known intracerebral aneurysm. } \\
\text { Respiratory events: deterioration } \\
\text { of the Horowitz index, } \\
\text { mechanical ventilation, hypoxia, } \\
\text { ARDS, acute pulmonary } \\
\text { dysfunction. } \\
\text { Hepatic events: liver failure or } \\
\text { liver dysfunction with an acute } \\
\text { increase in serum bilirubin from } \\
\text { baseline. } \\
\text { Haematological events not } \\
\text { related to anticoagulation } \\
\text { method: DIC, thrombocytosis. } \\
\text { SIRS criteria: tachypnoea, } \\
\text { hypopnoea, leucocytosis, } \\
\text { hypothermia, hyperthermia, } \\
\text { tachycardia or bradycardia. }\end{array}$ & $\begin{array}{l}\text { CVC-related adverse events: } \\
\text { - Haemorrhage at the site orCVC insertion with } \\
\text { requiring of transfusion }>1 \text { unit of packed red blood } \\
\text { cells and/or surgical intervention within } 12 \text { hours } \\
\text { following insertion. } \\
\text { - CVC-associated bloodstream infection (bacteraemia } \\
\text { and culture-positive confirmation of the same } \\
\text { organism from the dialysis catheter on removal). } \\
\text { - Ultrasonographically confirmed thrombus attributed } \\
\text { to CVC. } \\
\text { - Pneumothorax (for catheters placed in the internal } \\
\text { - jugular or subclavian position). } \\
\text { Haemothorax (for catheters placed in the internal } \\
\text { - } \text { Air embolism. } \\
\text { - Inadvertent arterial puncture at time of CVC } \\
\text { insertion. } \\
\text { RRT-associated hypotension: drop in blood pressure } \\
\text { requiring } \\
\text { - Initiation of vasopressor during RRT session. } \\
\text { - Need to escalate dose of vasopressor during RRT } \\
\text { - } \text { session. } \\
\text { - Premature discontinuation of RRT session. } \\
\text { - Any other intervention to stabilise blood pressure. } \\
\text { Severe hypophosphataemia }<0.5 \text { mmol/L. } \\
\text { New arrhythmia developed during dialysis and was not } \\
\text { present prior to dialysis: } \\
\text { - } \text { Atrial arrhythmia (excluding sinus arrhythmia or sinus } \\
\text { dialysis). }\end{array}$ & $\begin{array}{l}\text { Clinical results or typical } \\
\text { events in connection with } \\
\text { CVC or RRT, as defined } \\
\text { above, if the investigator } \\
\text { suspects a reasonable } \\
\text { causal relationship to the } \\
\text { investigational product. } \\
\text { All other serious adverse } \\
\text { events, regardless of } \\
\text { whether or not the } \\
\text { investigator suspects } \\
\text { a reasonable causal } \\
\text { relationship to the } \\
\text { investigational product. }\end{array}$ \\
\hline
\end{tabular}

ARDS, acute respiratory distress syndrome; CVC, central venous catheter; DIC, disseminated intravascular coagulation; SIRS, systemic inflammatory response syndrome.

corresponding to the relevant German definitions in the Good Clinical Practice (GCP) Ordinance (GCP-V). Adverse events and serious adverse events with a reasonable causal relationship to the investigated product, as defined in table 2, will be documented from the time of the first dose of heparin or citrate until discharge from the ICU. The following other adverse events independent of causal relationship will also be documented: (1) severe hypocalcaemia (ionised calcium $<0.9 \mathrm{mmol} / \mathrm{L}$ ), (2) allergic reaction during RRT (eg, heparin-induced thrombocytopaenia (HIT), thrombocytopaenia), (3) haemorrhage during dialysis, requiring transfusion of $>1$ unit of packed red blood cells, (4) organ failure due to other reasons than sepsis/ septic shock (eg, anaphylaxis, lung embolism), (5) onset of any other new sign, symptom or disease.

\section{Trial oversight}

The Department of Anesthesiology, Intensive Care and Pain Medicine of the University of Münster, Germany will serve as the central trial coordination centre. The data management will be coordinated by the centre of clinical trials in Leipzig, Germany. Both centres will work closely with the study sponsor and the clinical trials centre of Münster, Germany, to coordinate trial activities and will be responsible for developing electronic case report forms, training trial staff, performing data validation, monitoring activities, obtaining regulatory approvals and safety reporting.

The data safety monitoring board (DSMB) will monitor and review the randomisation process during the entire enrolment of the study: members are experts in critical care medicine, statistics and clinical research (online supplementary table 3). The task is to oversee the safety of the trial subjects in the clinical trial by periodically assessing the safety and effectiveness of the trial therapy, and to monitor the integrity and validity of the collected data and the conduct of the clinical trial. Throughout this process of surveillance, the DSMB provides the sponsor with recommendations regarding the continuation of the trial (eg, termination or modification) based on the collected data. 
The data necessary to fulfil this function are provided by the sponsor as determined by the DSMB. Among other datasets, these must include listings providing information on serious adverse events and further variables that the DSMB considers necessary at least every 6 months and when formal interim analysis is conducted.

\section{Ethics and dissemination}

The RICH trial has been approved by the Federal Institute for Drugs and Medical Devices (BfArM) (EudraCT-No.: 2014-004854-33), the leading Ethics Committee of the University of Münster (2016-648f-A) and the corresponding Ethics Committee at each participating site (online supplementary table 4 ). The results will be presented at national as well as international conferences in poster or oral presentations. The final manuscript will be published in a peer-reviewed journal and results will be used to update clinical practice guidelines on the management of RRT in AKI. ${ }^{16}$

The Standard Protocol Items: Recommendations for Interventional Trials checklist is included in online supplementary table 5 .

\section{CONCLUSION}

The RICH trial is a prospective, large randomised, multicentre, clinical trial with the aim to investigate whether RCA prolongs filter life span and overall survival in a 90-day follow-up period (two coprimary outcomes) as compared with SAH. Based on existing evidence, the KDIGO guidelines suggest using RCA for CRRT in patients with AKI in the absence of contraindications. However, the recommendation is classified as grade 2B, indicating that evidence is weak. Despite the guideline, a lot of practitioners do not use any anticoagulation strategy for CRRT at all ${ }^{17}$ although it has been shown that the filter life span is longer with the use of anticoagulants. ${ }^{18}$ This may be a consequence of the fear from potential side effects. The use of SAH is associated with increased bleeding risk, especially in surgical patients, and the development of HIT. RCA might lead to metabolic derangements and citrate accumulation. In addition, it has been shown that the use of anticoagulants influences the host immune response.

The RICH trial is the largest randomised study to prospectively answer the question which anticoagulation regimen in critically ill patients undergoing CRRT is associated with improved patient-centred outcomes. The results of this trial will improve the care of patients with AKI and allow to give more precise recommendations in future guidelines on AKI.

\section{TRIAL STATUS}

Recruitment was started in March 2016. We estimate to complete the study in March 2021.

\section{Author affiliations}

${ }^{1}$ Department of Anesthesiology, Intensive Care and Pain Medicine, University of Münster, Münster, Germany
${ }^{2}$ Department of Anesthesiology and Critical Care Medicine, Heinrich-Heine University of Düsseldorf, Düsseldorf, Germany

${ }^{3}$ Department of Intensive Care, University Medical Center Hamburg-Eppendorf, Hamburg, Germany

${ }^{4}$ Department of Nephrology, Charité University of Berlin, Berlin, Germany ${ }^{5}$ Department Intensive Care Medicine, University of Aachen, Aachen, Germany ${ }^{6}$ Institute of Biostatistics and Clinical Research, University of Münster, Münster, Germany

Acknowledgements The authors would like to thank all the participating centers and corresponding staff member involved in this trial.

Contributors MM conceived the study, helped design the trial and drafted the manuscript; MK performed the study and drafted the manuscript; JG helped design the trial and draft the manuscript; CW helped design the trial, performed study coordination and drafted the manuscript; DK-M helped design the trial and drafted the manuscript; SK helped design the trial and drafted the manuscript; TS helped design the trial and drafted the manuscript; GM helped design the trial and drafted the manuscript; $A Z$ conceived the study, designed the trial and drafted the manuscript. All authors read and approved the final manuscript.

Funding The trial is supported by the German Research Foundation (ZA 428/10-1). Competing interests None declared.

Patient consent for publication Not required.

Ethics approval Federal Institute for Drugs and Medical Devices, the leading Ethics Committee of the University of Münster and the corresponding Ethics Committee at each participating site.

Provenance and peer review Not commissioned; externally peer reviewed.

Open access This is an open access article distributed in accordance with the Creative Commons Attribution Non Commercial (CC BY-NC 4.0) license, which permits others to distribute, remix, adapt, build upon this work non-commercially, and license their derivative works on different terms, provided the original work is properly cited, appropriate credit is given, any changes made indicated, and the use is non-commercial. See: http://creativecommons.org/licenses/by-nc/4.0/.

\section{REFERENCES}

1. Hoste EA, Bagshaw SM, Bellomo R, et al. Epidemiology of acute kidney injury in critically ill patients: the multinational AKI-EPI study. Intensive Care Med 2015;41:1411-23.

2. Uchino S, Bellomo R, Morimatsu H, et al. External validation of severity scoring systems for acute renal failure using a multinational database. Crit Care Med 2005;33:1961-7.

3. Metnitz PGH, Krenn CG, Steltzer $\mathrm{H}$, et al. Effect of acute renal failure requiring renal replacement therapy on outcome in critically ill patients*. Crit Care Med 2002;30:2051-8.

4. Kellum JA, Angus DC. Patients are dying of acute renal failure * . Crit Care Med 2002;30:2156-7.

5. Gattas DJ, Rajbhandari D, Bradford C, et al. A randomized controlled trial of regional citrate versus regional heparin anticoagulation for continuous renal replacement therapy in critically III Adults. Crit Care Med 2015;43:1622-9.

6. Bai M, Zhou M, He L, et al. Citrate versus heparin anticoagulation for continuous renal replacement therapy: an updated meta-analysis of RCTs. Intensive Care Med 2015;41:2098-110.

7. Hetzel GR, Schmitz M, Wissing $\mathrm{H}$, et al. Regional citrate versus systemic heparin for anticoagulation in critically ill patients on continuous venovenous haemofiltration: a prospective randomized multicentre trial. Nephrol Dial Transplant 2011;26:232-9.

8. Oudemans-van Straaten HM, Bosman RJ, Koopmans M, et al. Citrate anticoagulation for continuous venovenous hemofiltration. Crit Care Med 2009;37:545-52.

9. Schilder L, Nurmohamed SA, Bosch FH, et al. Citrate anticoagulation versus systemic heparinisation in continuous venovenous hemofiltration in critically ill patients with acute kidney injury: a multicenter randomized clinical trial. Crit Care 2014;18:472.

10. Wu MY, Hsu YH, Bai CH, et al. Regional citrate versus heparin anticoagulation for continuous renal replacement therapy: a meta-analysis of randomized controlled trials. Am J Kidney Dis 2012;59:810-8.

11. Zhang Z, Hongying N. Efficacy and safety of regional citrate anticoagulation in critically ill patients undergoing continuous renal replacement therapy. Intensive Care Med 2012;38:20-8. 
12. Singer M, Deutschman CS, Seymour CW, et al. The Third International Consensus Definitions for Sepsis and Septic Shock (Sepsis-3). JAMA 2016;315:801-10.

13. Pocock SJ, Simon R. Sequential treatment assignment with balancing for prognostic factors in the controlled clinical trial. Biometrics 1975;31:103-15.

14. Uchino S, Bellomo R, Morimatsu $\mathrm{H}$, et al. Discontinuation of continuous renal replacement therapy: a post hoc analysis of a prospective multicenter observational study. Crit Care Med 2009;37:2576-82.
15. Bauer P. Multiple testing in clinical trials. Stat Med 1991;10:871-90.

16. Kellum J ALN, Aspelin P, Barsoum RS, et al. KDIGO clinical practice guideline for acte kidney injury 2012. Kidney Int Suppl 2012;2:1-138.

17. Thompson A, Li F, Gross AK. Considerations for medication management and anticoagulation during continuous renal replacement therapy. AACN Adv Crit Care 2017;28:51-63.

18. Brophy PD, Somers MJ, Baum MA, et al. Multi-centre evaluation of anticoagulation in patients receiving continuous renal replacement therapy (CRRT). Nephrol Dial Transplant 2005;20:1416-21. 